\title{
Differential conditioning as a function of cue presentation and $\mathrm{S}+$ extinction
}

\author{
ELIZABETH D. IVEY and STEPHEN F. DAVIS \\ Austin Peay State University, Clarksville, Tennessee 37040 \\ and \\ JOHN D. SEAGO \\ Concord College, Athens, West Virginia 24712
}

\begin{abstract}
Two groups of rats received differential instrumental conditioning trials in a two-phase experiment. One group received discriminative $S+/ S-$ cues prior to making the instrumental response. Such cues were presented to the second group upon inception of the response. During Phase 1 large or small reward was present on all trials. During Phase 2 (S+ extinction) reward was present only in the $S-$ (small reward) alternative. The results are seen as supportive of a frustration or inhibition interpretation. Discrepancies between the present and previous data are discussed.
\end{abstract}

In the area of differential conditioning, it is not uncommon to observe that performance to small reward ( $\mathrm{S}-$ ) is depressed, relative to that of subjects receiving only small reward, when large reward $(\mathrm{S}+)$ is concurrently received. Termed the "negative contrast effect" (NCE), this finding has been independently reported by several investigators (e.g., Bower, 1961; Ludvigson \& Gay, 1966, 1967).

Several factors have been found to influence performance to the $\mathrm{S}-$ alternative in this situation. For example, Ludvigson and Gay $(1966,1967)$ have found that presentation of discriminative $\mathrm{S}+/ \mathrm{S}-$ cues has a distinct influence on the NCE. If these cues were presented prior to the initiation of the instrumental response, then the NCE was substantially reduced. It should also be noted that these investigators reported that the largest NCEs occurred in conjunction with the initial presentation of the discriminative cues.

Additionally, McHose and Blackwell (1975) reported that the pattern of $S+$ partial reinforcement influenced performance to an S- alternative that was never reinforced. Consistent with earlier results (McHewitt \& McHose, 1970; McHose, McHewitt, \& Peters, 1972), McHose and Peters (1973) found that S- performance was inversely related to both amount and percentage of St reinforcement. Similarly, McHose and Howard (1973) found that frequency of S+ trials, relative to the total number of trials administered, was inversely related to S- speeds. Also, Harris, Collerain, Wolf, and Ludvigson (1970) reported the occurrence of a

This research was supported, in part, by a Tower Fund Research Grant from Austin Peay State University to the second author. Requests for reprints should be addressed to Stephen F. Davis, Department of Psychology, Austin Peay State University, Clarksville, Tennessee 37040.
NCE in a situation not requiring an instrumental running response to the $\mathrm{S}+$.

In several instances, a frustration and/or inhibition model has been employed to account for such results (e.g., Ludvigson \& Gay, 1967; McHose et al., 1972). However, other investigators have presented data supportive of absolute magnitude of reward (e.g., Goldstein \& Spence, 1963) and invariant rate of change in incentive motivation (Logan, 1968) interpretations. If the latter position is correct, then prior experimental and/or reinforcement history should have little or no effect upon performance.

The present two-phase study was designed to investigate the effects of extinction in the $\mathrm{S}+$ condition with and without discriminative cue presentation preceding the instrumental response. If performance is solely a matter of reward magnitude, then there should be no difference in performance between subjects presented the discriminative cues prior to making the response and those receiving such cues as the start door is raised (i.e., at the onset of the response). If, on the other hand, the discriminative cues (and their associated excitatory and inhibitory properties) do have an influence on performance, then differences might well be predicted.

\section{METHOD}
Subjects
Twenty female albino rats purchased from the Holtzman Company, Madison, Wisconsin, served as subjects. The subjects were approximately 90 days old at the beginning of pretraining. One week prior to the start of the experiment, subjects were placed on food deprivation and maintained at $85 \%$ free-feeding weight. All subjects were caged individually with water continuously available. 


\section{Apparatus}

The apparatus was similar to the one used by Ludvigson and Gay (1967). Basically, it consisted of two parallel runways (one black, one white), each $11.43 \mathrm{~cm}$ wide and $12.70 \mathrm{~cm}$ high. A $28.10-\mathrm{cm}$, gray movable startbox serviced both runways. Removable Masonite inserts, painted the same colors as the runways, were used to provide discriminative cues in the startbox. The startbox was separated from the $91.44-\mathrm{cm}$ run sections by a Masonite guillotine door. A second Masonite door separated the run section from a $30.48-\mathrm{cm}$ goalbox. Lifting the start door activated a Standard Electric timer; interrupting a photobeam $15.24 \mathrm{~cm}$ beyond the start door stopped the first timer (start time) and activated a second timer. Interrupting a second photobeam $76.20 \mathrm{~cm}$ beyond the first photobeam stopped the second timer (run time) and started a third timer. Interrupting a third photobeam located $5.08 \mathrm{~cm}$ in front of a recessed goal cup stopped the third timer (goal time). The entire apparatus was covered with $.64-\mathrm{cm}$ hardware cloth and was floored with wood.

\section{Procedure}

Prior to pretraining, the subjects were randomly assigned to two groups of 10 subjects each. Subjects in Group G started from the neutral gray (nondiscriminative) startbox, while subjects in Group BW were provided the discriminative cues in the startbox.

A pretraining period of 5 days preceded the start of the experiment. During the first 3 days, all subjects were handled and tamed for $1 \mathrm{~min}$ each, and pellet habituated to the $45-\mathrm{mg}$ Noyes reward pellets in the home cage. On each of the last 2 days of pretraining, each subject received a 5-min exploration period in the unbaited runway. One exploration period was allowed in each runway. Subjects in Group G were placed directly into the gray startbox to initiate each exploration period. For subjects in Group BW, the appropriately colored Masonite insert was in position when the subject was placed in the startbox.

Phase 1, acquisition, was begun immediately after pretraining. During this phase, all subjects received six trials (three $S+$, three $S-$ ) per day for 14 days (84 trials). The daily administration of $S+$ and $S$ - events was randomized with the restriction that no daily sequence could occur twice in a row. All subjects received Trial 1 before Trial 2 was administered, Trial 2 before Trial 3, and so on, with all subjects in a respective group receiving all six daily trials before the other group was run. The order for running groups alternated between days. Twelve 45-mg Noyes pellets were present on S+ trials, while one pellet was present on $S$ - trials. The designated of $S+$ and $S$ - with regard to the black and white runways was balanced within each group. To run a trial, the appropriate subject was removed from the home cage and placed into the startbox. Following a 5 -sec confinement period in the startbox, the start door was raised and the subject. was allowed to traverse the runway. The subject was removed from the goalbox upon consumption of the reward: Start, run, and goal latencies were recorded for each trial.

Phase 2 (S+ extinction) was begun immediately after Phase 1 and lasted 7 days (42 trials). The procedures used in Phase 1 were also in effect in Phase 2, the only exception being that no reward was present on any $S+$ trial.

\section{RESULTS}

The daily S+ and S- latencies for each subject were averaged to yield single $\mathrm{S}+$ and $\mathrm{S}-$ values. Prior to analysis, these averaged scores were reciprocated and, when multiplied by the appropriate constant, yielded speed scores in meters per second. Figures 1-3

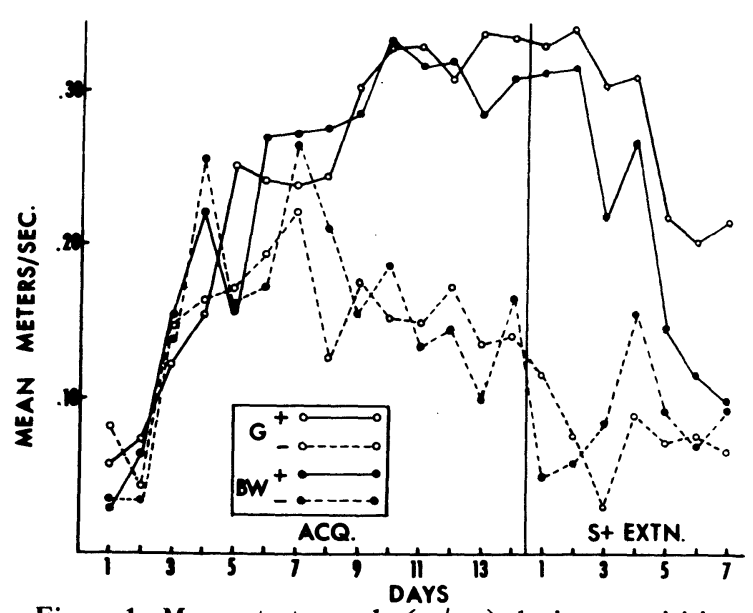

Figure 1. Mean start speeds $(\mathrm{m} / \mathrm{sec})$ during acquisition and S+ extinction.

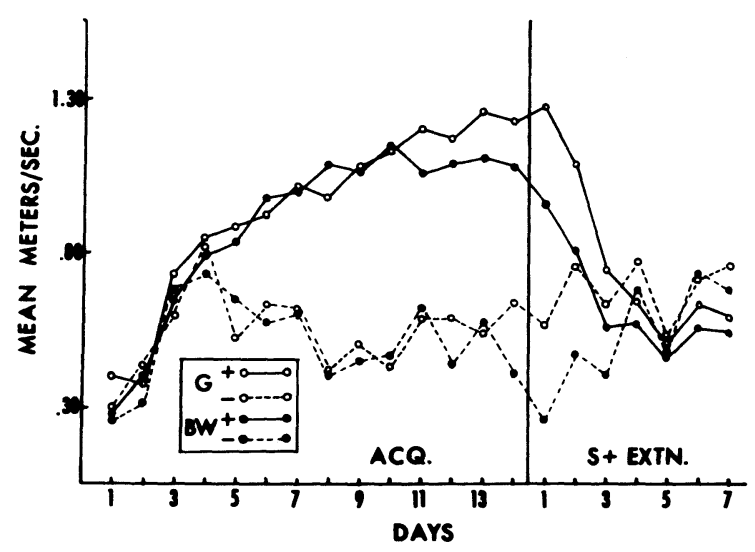

Figure 2. Mean run speeds $(\mathrm{m} / \mathrm{sec})$ during acquisition and S+ extinction.

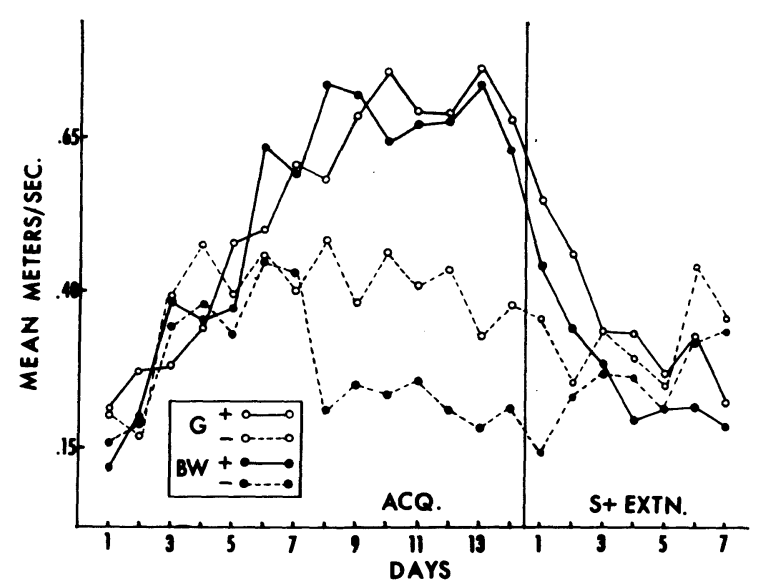

Figure 3. Mean goal speeds $(\mathrm{m} / \mathrm{sec})$ during acquisition and S+ extinction.

present the mean start, run, and goal speeds $(\mathrm{m} / \mathrm{sec})$, respectively, for the acquisition and $\mathrm{S}+$ extinction phases. Since many and somewhat different effects were obtained in the two phases, analyses for each phase will be presented separately. 


\section{Acquisition}

Analyses were performed over acquisition Days 9-14. These days represented the point at which discrimination had been achieved in all measures.

Start. Analysis of variance performed over the start measure speeds indicated that the $\mathrm{S}+$ vs. $\mathrm{S}-$ factor was significant $[F(1,18)=9.78, p<.01]$.

Run. Run measure analysis also yielded a significant $[F(1,18)=12.14, p<.01] S+$ vs. $S-$ effect.

Goal. Goal measure analysis yielded significance $[F(1,18)=10.64, p<.01]$ for the $S+$ vs. $S-$ factor and the Startbox Conditions ( $\mathrm{G}$ vs. BW) by $\mathrm{S}+/ \mathrm{S}-$ interaction $[F(1,18)=9.81, p<.01]$. The significant interaction was further probed through the use of Tukey's procedure and indicated that the $S$ - speeds for Group BW were significantly $(p<.01)$ depressed below the S- speeds of Group G.

\section{S+ Extinction}

Start. Start measure analysis yielded significance for the $S+$ vs. $S-[F(1,18)=8.53, p<.01]$ and days $[F(6,108)=2.95, p<.05]$ factors and the Startbox Conditions by $S+/ S-$ interaction $[F(1,18)=9.07$, $p<.01]$. Tukey's procedure was used to probe the significant interaction and indicated that $\mathrm{S}+$ speeds for Group BW were significantly slower $(p<.01)$ than $\mathrm{S}+$ speeds for Group G.

Run. Analysis of the run speeds indicated the following significant effects: days $[F(6,108)=2.79, p<.05]$, the $\mathrm{S}+\mathrm{S}-$ by Days interaction $[\mathrm{F}(6,108)=2.35$, $\mathrm{p}<.05]$, and the Startbox Conditions (G vs. BW) by $\mathrm{S}+/ \mathrm{S}-$ by Days interaction $[\mathrm{F}(6,108)=2.41, \mathrm{p}<.05]$. Further analysis (Tukey's procedure) of the significant triple interaction indicated that at Day $1, \mathrm{~S}+$ speeds for Group $G$ were significantly $(p<.05)$ faster than S- speeds for Group BW. On Day 2, St speeds for Group $G$ remained significantly $(p<.05)$ faster than all other conditions. Speeds ( $\mathrm{S}+$ ) for Group BW and $S$ - speeds for Group $G$ were significantly $(p<.05)$ faster than S- speeds for Group BW, but did not differ from each other. The only other significant difference occurred on Day 3, when St speeds for Group G were significantly $(p<.05)$ faster than $S-$ speeds for Group BW.

Goal. Goal speed analysis indicated that the days $[F(6,108)=2.63, p<.05], S+/ S-$ by Days interaction $[F(6,108)=2.63, p<.05]$, and Startbox Conditions ( $\mathrm{G}$ vs. BW) by $\mathrm{S}+/ \mathrm{S}-$ by Days interaction $[F(6,108)=2.89, \quad p<.05]$ effects were significant. Inspection of the significant triple interaction (Tukey's procedure) indicated that at Day $1, \mathrm{~S}+$ speeds for Group $G$ were significantly $(p<.05)$ faster than all other conditions. Also, Group G $\mathrm{S}$ - speeds were significantly $(\mathrm{p}<.05)$ faster than Group BW S+ speeds. On Day 2, Group G S+ speeds were significantly $(p<.05)$ faster than all other conditions. On Days 4 and 6 , Group G S+ speeds were significantly $(\mathrm{p}<.05)$ faster than Group BW S+ speeds. On Day 6, Group G $\mathrm{S}$ - speeds were significantly $(\mathrm{p}<.05)$ faster than Group BW S+ speeds. On Day 7, S- speeds for both Groups BW and $G$ were significantly $(p<.05)$ faster than S+ speeds for Group BW.

\section{DISCUSSION}

Since both groups received identical rewards administered under identical schedules, the occurrence of $\mathrm{S}+$ and $\mathrm{S}-$ differences during acquisition and extinction indicates that sheer magnitude of reward does not appear to be the sole factor involved in determining performance in this situation. Hence, a consideration of other factors, such as cue presentation in the startbox, certainly seems appropriate.

If the results of the present study could be viewed in isolation, then an interpretation might be relatively straightforward. However, when these data are viewed in the context of previously reported differential conditioning studies and theory, interpretation problems become all too apparent. The major discrepancy appears to center around acquisition performance, especially $\mathrm{S}-$ speeds. It will be recalled that the studies reported by Ludvigson and Gay $(1966,1967)$ presented relevant $S+$ and $S$ - cues in the startbox prior to the initiation of the instrumental response. In those studies, preresponse cue presentation resulted in a lessening of the difference between $\mathrm{S}+$ and $\mathrm{S}-$ speeds, relative to a group receiving the discriminative cues only at the initiation of the instrumental response. This effect appeared to be due primarily to less severely depressed $S$ - speeds. Further, these studies indicated that the difference between $\mathrm{S}+$ and $\mathrm{S}$ - speeds decreased as the subjects approached the goal. The latter result was noted in all groups and did not appear to be a function of whether discriminative cues were presented prior to initiation of the instrumental response or not.

Since a group (Group BW) receiving preresponse exposure to discriminative $S+$ and $S$ - cues was included in the present study, it was anticipated that similar results would be shown. As can be seen from Figure 1, this was certainly not the case. Large $\mathrm{S}+/ \mathrm{S}$ - start measure differences were shown by both groups.

Additionally, it was anticipated that the $\mathrm{S}+/ \mathrm{S}$ - differences for both Groups BW and $G$ would dissipate as the subjects approached the goal. Figures 2 and 3 indicate that this did not happen. If anything, $\mathrm{S}+/ \mathrm{S}-$ differences became more pronounced for Group BW in the goal measure.

How should these discrepancies be resolved? One possible solution may lie in a consideration of possible time-in-startbox differences. In the Ludvigson and Gay (1967) study, after being placed in the startbox, all subjects were required to orient toward the start door for 1 sec before the door was raised and the instrumental response initiated. As a relatively short (5-sec) startbox confinement period was imposed on all subjects in the present study, it appears quite likely that their confinement was considerably shorter than that imposed by Ludvigson and Gay (1967). Concerning a specific mechanism underlying the present data, it is tempting to speculate that the shorter confinement "primed" the subjects in Group BW to make an inhibitory response. However, due to the short confinement, the full magnitude of this partially aroused inhibition was not manifested until after the instrumental response had begun and the subject actually approached the goal.

A similar line of reasoning could be applied to the performance of Group $G$ in the present study. As mentioned, this group did not show the decrease, predicted from the Ludvigson and Gay (1967) data, in S+/S- differences as they approached the goalbox. Time-in-startbox differences may, once again, provide an answer for these discrepant results. Assuming that the sight of the neutral gray startbox did arouse 
some (albeit nonspecific) inhibition or frustration, then it might further be suggested that Group G subjects in the present study were not permitted sufficient time for this inhibition to dissipate. Sufficient time for the dissipation of inhibition was apparently permitted in the Ludvigson and Gay (1967) study.

\section{REFERENCES}

Bower, G. H. A contrast effect in differential conditioning. Journal of Experimental Psychology, 1961, 62, 196-199.

Davis, S. F., Gilbert, R. F., \& Seaver, W. E., III. Stimulus onset and its effect on $S+$ and $S$ - performance in differential conditioning. Psychonomic Science, 1971, 25, 29-31.

Goldstein, H., \& Spence, K. W. Performance in differential conditioning as a function of variation in magnitude of reward. Journal of Experimental Psychology, 1963, 65, 86-93.

Harris, D. R., Collerain, I., Wolf, J. C., \& Ludvigson, H. W. Negative $S$ - contrast with minimally contingent large reward as a function of trial initiation procedure. Psychonomic Science, 1970, 19, 189-190.

LogAN, F. A. Incentive theory and changes in reward. In K. W. Spence \& J. T. Spence (Eds.), The psychology of learning and motivation. New York: Academic Press, 1968. Pp. 1-30.

Ludvigson, H. W., \& GAY, S. E. Differential reward conditioning: S- contrast as a function of the magnitude of $\mathrm{S}+$. Psychonomic Science, 1966, 5, 289-290.
Ludvigson, H. W., \& GAY, R. A. An investigation of conditions determining contrast effects in differential reward conditioning. Journal of Experimental Psychology, 1967, 75, 37-42.

McHewitT, E. R., \& McHose, J. H. Role of nonreward in differential conditioning. Journal of Experimental Psychology, 1970, 84, 531-534.

McHose, J. H., \& Blackwell, D. R. Performance in differential instrumental conditioning as a function of the pattern of partial S+ reward. Animal Learning \& Behavior, $1975,3,63-66$.

McHose, J. H., \& How ARD, G. S. Performance in differential instrumental conditioning with infrequent $S+$ presentations. Bulletin of the Psychonomic Society, 1973, 1, 132-134.

McHose, J. H., Mchewit,, E. R., \& Peters, D. P. Average reward as a determinant of S- performance in differential conditioning. Psychonomic Science, 1972, 29, 129-132.

McHose, J. H., \& Peters, D. P. Differential instrumental conditioning as a function of percentage and amount of positive stimulus reward. Journal of Experimental Psychology, 1973, 100, 413-415.

(Received for publication December 27, 1977.) 